\title{
Image Segmentation Using Curve Evolution and Anisotropic Diffusion: An Integrated Approach
}

\author{
Yongsheng Pan J. Douglas Birdwell Seddik M. Djouadi \\ Laboratory for Information Technologies \\ Department of ECE \\ University of Tennessee \\ Knoxville, TN, 37996 \\ ypan@lit.net \\ birdwell@lit.net \\ djouadi@ece.utk.edu
}

\begin{abstract}
In this paper, a new model is proposed for image segmentation that integrates the curve evolution and anisotropic diffusion methods. The curve evolution method, utilizing both gradient and region information, segments an image into multiple regions. During the evolution of the curve, anisotropic diffusion is adaptively applied to the image to remove noise while preserving boundary information. Coupled partial differential equations (PDE's) are used to implement the method. Experimental results show that the proposed model is successful for complex images with high noise.
\end{abstract}

\section{Introduction}

Active contour models are widely used in image segmentation problems. In these models, curves are evolved in an image from initial locations, in response to information derived from the image, to detect object boundaries. These methods are derived using variational methods, and are implemented using finite difference approximations to PDE's and level sets.

The geometric model of active contours, proposed by Caselles et al. [1] and Malladi et al. [2], evolves curves in a Eulerian formulation and is implemented via level set algorithms [3][4]. This model, compared to the classic parametric model put forward by Kass et al. [5], has several advantages, mainly the capacity to automatically handle topological changes and simultaneously detect multiple objects. Several models [6]-[13] have been proposed that follow this approach, and good results have been achieved.

Several problems, however, remain. First, a boundary leakage problem exists for some models [6][7], in which the evolving curve does not stop at the boundaries of objects. Second, most of these models utilize either gradient information [6] or region information [8][9] alone. Both gradient and region information are used in some models [7][10][12], but the information is not fully utilized [7], or several assumptions are required [10][12]. Finally, methods are needed that are less sensitive to noise.

A new geometric active contour model is proposed in this paper that integrates curve evolution and anisotropic diffusion. The curve evolution method utilizes both gradient and region information for image segmentation. This model solves the boundary leakage problem. It is also able to deal with complicated cases such as color images and images with triple junctions. Anisotropic diffusion methods are integrated in the proposed model to reduce the effects of noise without smoothing the boundaries of deteced segments.

The paper is organized as follows. In section 2, previous work on both curve evolution and anisotropic diffusion is reviewed. Details of the proposed model are described in section 3. Section 4 discusses implementation issues. In section 5, experimental results are given and analyzed. A summary is provided in section 6 .

\section{Previous Work}

This section provides a short review of previous work on curve evolution and anisotropic diffusion. Problems associated with both methods will be analyzed, providing a foundation and justification for the proposed model in this paper. 


\subsection{Curve Evolution}

In several of the active contour models mentioned above, either gradient information [6] or region information [8][9] alone is utilized to perform image segmentation. Some models [7][10][12] use both types of information, but problems and limitations still exist.

For earlier models, gradient information alone is usually used to stop a curve at boundaries of objects, by weighting the evolution speed using a monotone decreasing function of image gradients [6]. Kichenassamy et al. [6] proposed minimization of the following energy functional:

$$
L_{\phi}(t)=\int_{0}^{1}\left\|\frac{\partial C}{\partial p}\right\| \phi d p
$$

where $C$ is the evolving curve, $p$ is the parameter of the curve $C$, and $\phi$ is a weighting function that depends upon image content. The energy functional measures the weighted length of $C$. The minimization of this energy functional leads to the following curve evolution model:

$$
\psi_{t}=\phi(x, y)\left(v+\epsilon \cdot \operatorname{div}\left(\frac{\nabla \psi}{|\nabla \psi|}\right)\right)|\nabla \psi|+\nabla \phi \cdot \nabla \psi
$$

where $\psi$ is the level set of the evolving curve $C$, which means $C=\{(x, y): \psi(x, y)=0\}$. div stands for the divergence operator, $v$ represents the speed of curve evolution, and $\epsilon$ is a positive constant. $\phi(x, y)$ acts as a "stopping term" using gradient information. $\phi$ takes the form $1 /\left(1+\left|\nabla G_{\sigma} * I\right|\right)$, where $G_{\sigma}$ represents the Gaussian kernel with variance $\sigma$, and $I$ is the image function.

Although this model works well in many cases, it allows boundary leakage when image gradients are not very high. Siddiqi et al. [7] combines gradient and area information for shape segmentation. Their methods have good performance, but boundary leakage still exists.

In the papers of Chan and Vese [8], region information is utilized for image segmentation. In their methods, the segmentation problem is formulated as the minimization of an energy functional simplified from the Mumford-Shah model [15]. The energy functional, which is also called the minimal variance criterion [13], takes the form:

$$
\begin{aligned}
F\left(c_{1}, c_{2}, C\right) & =\mu \cdot \operatorname{Length}(C) \\
& +\lambda_{1} \iint_{\text {inside }(C)}\left|I(x, y)-c_{1}\right|^{2} d x d y \\
& +\lambda_{2} \iint_{\text {outside }(C)}\left|I(x, y)-c_{2}\right|^{2} d x d y
\end{aligned}
$$

where $I$ is the original image, $C$ is the evolving curve, and $c_{1}$ and $c_{2}$ are selected as the average value of pixels inside and outside $C$, respectively. $\mu, \lambda_{1}$ and $\lambda_{2}$ are positive constants.
This model is implemented using level set methods and provides very good performance for bimodal images. Vese et al. later extended the model to multi-modal images [9]. In their model, however, gradient information is not utilized. Tsai et al. [10] implemented the Mumford-Shah model [15] using curve evolution methods and extended it to image noise reduction, interpolation and magnification.

Paragios et al. [11] put forward a model of coupled active regions to utilize both gradient and region information in a statistical framework. In this model, assumptions on the statistics of pixels and regions in the image are made, which impose limits on the model's performance.

Xie et al. [12] proposed a region-aided geometric snake model, which also makes use of the combination of gradient and region information. The method resolves the boundary leakage problem by using the region information. However, it seems difficult to deal with textured images by applying this method together with the tensor measure.

Kimmel [13] presents a general model that incorporates the alignment term, the geodesic active contour model [14] and the minimal variance criterion [8]. The energy functional to be minimized takes the following form:

$$
\begin{aligned}
E\left(C, c_{1}, c_{2}\right) & =-\oint_{C}|\langle\nabla I, \vec{n}\rangle| d s+\alpha \oint_{C} g(C(s)) d s \\
& +\beta \frac{1}{2}\left(\iint_{\text {inside }(C)}\left(I-c_{1}\right)^{2} d x d y\right. \\
& \left.+\iint_{\text {outside }(C)}\left(I-c_{2}\right)^{2} d x d y\right)
\end{aligned}
$$

In this way, both gradient and region information are utilized for image segmentation.

Another issue for active contour models is that noise should be carefully processed. For models using region information, image denoising is an independent step. Gaussian filters are usually used to smooth the image. For those models using gradient information, a Gaussian smoothing method is selected to reduce the effects of noise when calculating the gradients. In this process, however, the boundaries are also smoothed, which makes it difficult to find the boundary locations and encourages boundary leakage. In the present paper, anisotropic diffusion methods are used to address this problem and are introduced below.

\subsection{Anisotropic Diffusion}

Anisotropic diffusion is a good method for image denoising. It prefers intra-region smoothing to inter-region smoothing, and is thus able to smooth the noise while keeping the boundaries from being smoothed.

Anisotropic diffusion was first introduced by Perona and Malik [16]. The basic idea is to evolve a family of smoothed images $I(t)$ from an initial image $I_{0}$ using the following 
partial differential equation

$$
I_{t}=\operatorname{div}(g(|\nabla I|) \nabla I)=g(|\nabla I|) \triangle I+\nabla g \cdot \nabla I
$$

where $g(|\nabla I|)$ is designed to preferably smooth pixels inside a region rather than pixels near the boundary. $g(|\nabla I|)$ is usually selected as:

$$
g(|\nabla I|)=\exp \left(-(|\nabla I| / K)^{2}\right)
$$

or

$$
g(|\nabla I|)=\frac{1}{1+(|\nabla I| / K)^{2}}
$$

where $K$ is a constant that is tuned for a particular application.

In [17], You et al. showed that the Perona-Malik anisotropic diffusion listed above is equivalent to minimizing the following functional using a gradient descent method:

$$
E(I)=\iint_{\Omega} \frac{\nabla I}{1+(|\nabla I| / K)^{2}} d x d y
$$

This paper also shows that the Perona-Malik anisotropic diffusion method is ill-posed in the sense that images close to each other are likely to diverge during the diffusion process. Another problem with this method is the formation of density steps near boundaries, termed staircasing [18].

A theoretical analysis of the Perona-Malik method has shown that a weak solution is not guaranteed to exist [19]. In practice, however, this method usually performs very well. Weickert [20] and You [21] have examined the differences between continuous and discrete anisotropic diffusion. They show that discrete anisotropic diffusion is wellposed even if its continuous counterpart is ill-posed. The performance of discrete anisotropic diffusion, however, depends on finite difference schemes and grid sizes.

Several methods have been proposed to solve these problems. Alvarez et al. [22] proposed a well-posed method to selectively smooth the pixels in the image in its tangential direction

$$
I_{t}=g(G *|\nabla I|)|\nabla I| \operatorname{div}\left(\frac{\nabla I}{|\nabla I|}\right)
$$

where $G$ is a Gaussian kernel and $G *|\nabla I|$ is a local estimate of $\nabla I$ for noise elimination. This method, together with the Perona-Malik method, makes use of local information only, which provides poor estimation of the gradient and its direction. Global information should be used to improve these models, such as the Gabor method in [23]. Many models have been proposed recently to improve the diffusion performance, such as [24], [10], [25] and [26].

\section{Integration of Curve Evolution and Anisotropic Diffusion}

In this section, a new active contour model is proposed, which integrates curve evolution and anisotropic diffusion. The proposed curve evolution model, which makes use of both gradient and region information in the image, will be introduced first. Then, the connections between curve evolution and anisotropic diffusion are examined. Finally, the integration of curve evolution and anisotropic diffusion is shown. In the proposed model, anisotropic diffusion is selectively applied to images to reduce the effects of noise during curve evolution. Curve Evolution and anisotropic diffusion are combined by coupled PDE's.

\subsection{Curve Evolution Model}

The proposed model begins with an energy functional that integrates gradient and region information using the form

$$
\begin{aligned}
E(C) & =\alpha \cdot \int_{0}^{1}\left\|\frac{\partial C}{\partial p}\right\| \phi d p \\
& +(1-\alpha) \lambda \iint_{\text {inside }(c)}\left|I(x, y)-c_{1}\right|^{2} d x d y \\
& +(1-\alpha) \lambda \iint_{\text {outside }(c)}\left|I(x, y)-c_{2}\right|^{2} d x d y
\end{aligned}
$$

The minimization of (10) leads to the following active contour model

$$
\begin{aligned}
\psi_{t} & =\alpha \phi_{l}(v+\epsilon \kappa)|\nabla \psi|+\nabla \phi_{l} \cdot \nabla \psi \\
& +(1-\alpha) \lambda \delta_{\beta}(\psi)\left[\left(I-c_{2}\right)^{2}-\left(I-c_{1}\right)^{2}\right]
\end{aligned}
$$

In both equations, the weight function $\phi_{l}$ is selected as $1 /\left(1+|\nabla I|^{2}\right)$. For more details about the derivation, refer to [6][7][13].

The first term in the right hand side of (11) is a combination of constant motion and curvature motion. In this term, $v$ is the inflationary term, which attracts the curve in one direction, either expanding or shrinking; $\kappa$ represents the curvature, which keeps the curve smooth when evolving. The third term makes use of the region information, which helps to stop the curve at the boundaries of interest. In this term, $\delta_{\beta}(x)=\beta /\left(\pi\left(x^{2}+\beta^{2}\right)\right)$ acts like an approximate delta function, as in [8]. $\alpha, \beta$ and $\epsilon$ are positive constant coefficients. $\lambda$ is a normalization constant to make the values of gradient and region information comparable. $\alpha$ lies between 0 and 1 and determines the weights of the gradient and the region information. $\alpha$ is usually set to 0.5 . For those images where weak edges exist, $\alpha$ is set to be less than 0.5 to increase the weight of the region information; 0.2 is usually a good choice for $\alpha$ in such cases. 
When $\alpha$ is set to 1 , only gradient information is used, and the model is the same as model (1) in [6]. When the function $\phi$ is chosen to 1 everywhere and $v$ is set to $0,(11)$ becomes model (3) in [8]. Another point worth mentioning is that the minimization of the energy functional (10), according to the proofs in [13], will lead to the following active contour model

$$
\begin{aligned}
\psi_{t} & =\alpha \phi_{l}(v+\epsilon \kappa)|\nabla \psi|+\nabla \phi \cdot \nabla \psi \\
& +(1-\alpha) \lambda \delta_{\beta}(\psi)\left[\left(I-c_{2}\right)^{2}-\left(I-c_{1}\right)^{2}\right]|\nabla \psi|
\end{aligned}
$$

Note that the region information, in (12), influences the speed of the evolving curve in its normal direction. Our model in (11) constructs a region force field to guide the curve evolution. The region force field bears some similarity to the region force diffusion model in [12], but differs in that our region force field varies with the evolving curve.

Utilization of region information as shown above is particularly suitable for a bimodal image. To deal with images containing multiple objects, a hierarchical approach is proposed, which is similar to the one in [10]. In this approach, an image is segmented into two sub-images, and then the method is recursively applied on these sub-images. The recursion continues until each region contains only one object. In this way, the number of regions in the image need not to be known a priori nor estimated in the segmentation [11]. We only need to detect whether each of the subimages contains more than one object, which can be accomplished using the histogram of the sub-image.

The proposed method is easy to extend to vector-valued images. For a vector-valued image $I: \mathcal{R}^{2} \rightarrow \mathcal{R}^{m}$, the length weight function is selected as:

$$
\phi_{l}=\frac{1}{m} \sum_{i=1}^{m} \frac{1}{1+\left\|\nabla G_{\sigma} * I_{i}\right\|^{n}}
$$

Accordingly, the region weight function is selected so that the level set function takes the following form:

$$
\begin{aligned}
\psi_{t} & =\alpha \phi_{l}(v+\epsilon \kappa)|\nabla \psi|+\nabla \phi \cdot \nabla \psi \\
& +(1-\alpha) \delta_{\beta}(\psi) \frac{1}{m}\left[\sum_{i=1}^{m}\left(I_{i}-c_{2 i}\right)^{2}-\left(I_{i}-c_{1 i}\right)^{2}\right]
\end{aligned}
$$

For color images, three channels of information can be directly used in the above formulas. For textured images, preprocessing techniques, such as wavelet or Gabor transforms, can be performed, and the resulting vector-valued images are used.

\subsection{Connections between Curve Evolution and Anisotropic Diffusion}

Connections between curve evolution methods and anisotropic diffusion methods are examined before introducing anisotropic diffusion to the proposed model.
For the curve evolution model in (2), the term $\operatorname{div}\left(\frac{\nabla \psi}{|\nabla \psi|}\right)$ corresponds to the curvature of the evolving curve. It is used to keep the evolving curve smooth. The constant $\epsilon$ is usually chosen much smaller than $v$. This term can then be neglected in the following analysis, without affecting the conclusion. Then (2) becomes

$$
\psi_{t}=v \phi(x, y)|\nabla \psi|
$$

Since $\phi(x, y)$ is related to the image only and independent of the evolving curve, the above equation is equivalent to the minimization of the following functional via Green's theorem [7] (set $v=1$ here):

$$
E(I)=\iint_{\Omega} \phi(x, y) d x d y=\iint_{\Omega} \frac{1}{1+\left|\nabla G_{\sigma} * I\right|} d x d y
$$

Comparing the energy functional of curve evolution (16), with that of anisotropic diffusion (8), we find that they have structral similarities.

This similarity shows the relationship between curve evolution and anisotropic diffusion, which gives a clue to their combination. Curve evolution methods (3)(4)(10) minimize the energy functional by evolving a simple closed curve, which corresponds to the zero level set of $\psi$. They make use of global information and smoothing to avoid staircasing. Anisotropic diffusion methods (5)(9), on the other hand, minimize the energy functional by smoothing the whole image, which can be viewed as a set of curves corresponding to multiple level sets. They utilize the local information and reduce the effects of noise. Thus, the combination of these methods should solve the above problems and provide good performance. The method of coupled PDE's [27] is a good candidate for the combination.

\subsection{Curve Evolution Model integrating Anisotropic Diffusion}

Anisotropic diffusion methods are utilized to reduce the effects of noise on (11). Since both gradient and region information are used in the model, anisotropic diffusion methods are separately applied for each of them.

For gradient information, the Perona-Malik method (5) is applied, in which $g(|\nabla I|)=\frac{1}{1+(|\nabla I| / K)^{2}}$. Backward diffusion in the method (5) strengthens the edge gradient, which helps to stop the curve at object boundaries. Region information in the curve evolution model, on the other hand, helps to reduce staircasing. Since curve evolution is used to minimize the energy functional, only closed contours exist in the final result for closed initial contour, and staircasing problems are avoided. During curve evolution, only the gradient information near the evolving curve is used. PeronaMalik anisotropic diffusion, therefore, is applied to a narrow band close to the evolving curve. 
For region information, the method (9) in [22] is applied to the regions away from the evolving curve. Since this method is well-posed and no backward diffusion is introduced, noise in the image will be effectively smoothed, and region information will be fully utilized.

The proposed model is described by the coupled PDE's:

$$
I_{t}=f(\psi) \operatorname{div}\{g(|\nabla I|) \nabla I\}+(1-f(\psi)) g(|\nabla I|)|\nabla I| \operatorname{div} \frac{\nabla I}{|\nabla I|}
$$

$\psi_{t}=\alpha \phi_{l}(v+\epsilon \kappa)|\nabla \psi|+(1-\alpha) \delta_{\beta}(\psi)\left[\left(I-c_{2}\right)^{2}-\left(I-c_{1}\right)^{2}\right]$

where $f(\psi)$ acts as an indicator function and takes the following form:

$$
f(\psi)= \begin{cases}1 & \text { if the pixel is close to } \psi \\ 0 & \text { if the pixel is far away from } \psi\end{cases}
$$

In the proposed model, curve evolution methods help anisotropic diffusion methods by reducing staircasing, while anisotropic diffusion methods help the curve evolution methods by reducing noise without smoothing object boundaries. This concept is implemented by introducing the coupling between the two PDE's (17) and (18).

\section{Implementation Issues}

The proposed model is implemented using level set methods [3][4]. The narrow-band methods in [3] are utilized to reduce the computational load, and the fast marching methods are applied for reinitialization. The narrowband is defined to contain those pixels which are no more than 6 pixels away from the evolving curve. In the implementation, the speed of the evolving curve $v$ is set to make the curve shrink at speed 1 . The coefficient of the curvature $\epsilon$ is set to be 0.5 .

For anisotropic diffusion, the Perona-Malik method (5) is applied to the pixels in the narrow-band, and the selective diffusion method (9) is applied elsewhere in the image. Each of the diffusion methods is applied for 20 iterations during initialization and reinitialization. The threshold $K$ in the Perona-Malik method is set to be 40 unless specifically mentioned.

Another implementation issue is the choice for the value of the normalization parameter $\lambda$ in (18) for specific images. To reduce the labor to empirically choosing its value, a heuristic method is utilized in the implementation. Since the maximal value of the gradient function $\phi$ is $1.0, \lambda$ is chosen as the maximal absolute value in the region force field. In this way, the largest absolute value of the region term is also 1.0, making it comparable to the gradient information. Experimental results show that this implementation achieves very good experimental results.
In this approach, the parameter $\lambda$ is no longer a constant and varies with the evolving curve in each iteration. Thus, the implementation changes to some extent the energy functional to be minimized (10). Convergence problems may be introduced. Adaptive control techniques may be utilized here, which is a topic for ongoing research.

\section{Experimental Results}

Experimental results from the proposed model are given in this section. The program is implemented on a computer which has two Intel(R) Xeon ${ }^{T M} 2.4 \mathrm{GHz}$ CPUs , $2 \mathrm{G}$ bytes RAM, and runs the Red Hat Linux operating system. The CPU times given in this paper are the sums of system CPU times and user CPU times. The system CPU time is usually very small, typically $0.01-0.03$ second.

Fig. 1 illustrates the role of region information in the proposed model. The initial curve in the image is set as a small circle in its center (a). The curve is set to shrink, but the region information forces the curve to move and expand to the correct boundary of the object (c). The final result is very good, even though the image is very noisy. The region information also helps to stop the evolving curve at the correct position and avoid the boundary leakage problem. Images (b) and (d) show the region force field at the start and end of the curve evolution. Note that no big differences exist between (b) and (d).

Fig. 2 shows the segmentation of multiple objects in an image. Note that the original image (a) is much noisier than Fig. 1(a). Images (b), (c) and (d) in Fig. 2 show the evolving curve for the proposed model. The irregularity in the boundary of the big circle occurs in (d) because the corresponding area is more similar to the background than the circle. The objects are successfully segmented in spite of the noise. This experiment shows that anisotropic diffusion methods used by the proposed model work very well to reduce the effects of noise.

In Fig. 3, comparisons between the proposed method and the methods of [6] and [8] are given when $\alpha$ is set to be 0.1 . The original image (a) is generated by smoothing an ideal circle using a 13-by-13 Gaussian filter. It can be seen that our method (b) and the method in [8] (c) correctly segment out the object in the image. The results from (b) and (c) show no big differences. In comparison, the method in [6] (d) allows the boundary leakage. This example shows that our model performs very well for images with weak edges.

Fig. 4 shows the segmentation results of a real image with initial contour (a1) and (a2). Images (b1)-(d1) show the results of the proposed method, in which the rock is successfully segmented. (b2)-(d2) show those of the method in [8]. The evolving curve, driven mostly by the region information, doesn't stop at the boundaries of the rock. It can be 
clearly seen that in this case our results (d1) are much better than (d2) produced by the method of [8]. Fig. 5 shows more experimental results on real images.

The results in Fig. 6 show the segmentation of a triple junction in an image, in which a hierarchical approach is utilized. For the original image (a), the first iteration of the model separates the red region from the green and blue regions. The second iteration divides the green region and the blue region. The triple junction is successfully segmented by these two iterations.

In Fig. 7, the susceptibility of the model to the staircasing problem is tested. For the original image Fig. 7(a), which is the same as Fig. 1(a), Perona-Malik diffusion is performed with the threshold $K=10$. Staircasing occurs in (b) as expected. (c) magnifies a portion of (b), which shows staircasing. (d)-(g) show the results of the proposed model. The final segmentation result in (g) is good. (h) also magnifies part of (g). It can be seen that staircasing is not present, and the image is segmented into two regions.

In Fig. 8, the proposed method is applied to an image with heavy noise, as can be seen in the original image (a). (b) and (c) show intermediate segmentation results. (d) contains the final segmentation results. From these results, we can see that our model achieves acceptable results in the presence of high noise.

\section{Summary}

A new image segmentation method is proposed in this paper, which integrates curve evolution and anisotropic diffusion. The curve evolution method, utilizing both the region and gradient information, solves the boundary leakage problem. It can segment complicated images with the hierarchical approach. Anisotropic diffusion methods reduce the effects of noise on both the gradient and the region information. Experimental results show that the model works very well. Further research will be focused on the extension of the proposed model for the segmentation of textured images.

\section{References}

[1] V. Caselles, F. Catte, T. Coll, and F. Dibos, "A geometric model for active contours," Numerische Mathematik, V. 66, pp. 1-31, 1993.

[2] R. Malladi, J. Sethian, and B. Vemuri, "Evolutionary fronts for topology independent shape modeling and recovery," Proc. 3rd Eur. Conf. Computer Vision, pp. 313, 1994.

[3] J. Sethian, Level Set Methods: Evolving Interfaces in Geometry, Fluid Mechanics, Computer Vision, and Materials Science Cambridge U. Press, 1999.
[4] S. Osher and J. Sethian, "Front propagating with curvature-dependent speed: algorithms based on Hamilton-Jacobi formulations," J. Compu. Phys., V. 79, pp. 12-49, 1988.

[5] M. Kass, A. Witkin, and D. Terzopoulos, "Snakes: Active contour models," Int. J. Comput. Vis., V. 1, pp. 321331, 1988.

[6] S. Kichenassamy, A. Kumar, P. Olver, A. Tannenbaum, and A. Yezzi, "Gradient flows and geometric active contour models," Fifth Int. Conf. Computer Vision, pp. 810-815, 1995.

[7] K. Siddiqi, Y. Lauziere, A. Tannenbaum, and S. Zucker, "Area and length minimizing flows for shape segmentation," IEEE Trans. Image Processing, V. 7, no. 3, pp. 433-443, Mar. 1998.

[8] T. Chan, and L. Vese, "Active contours without edges," IEEE Trans. Image Processing , V. 10, no. 2, pp. 266277, Feb. 2001.

[9] L. Vese and T. Chan, "A multiphase level set framework for image segmentation using the Mumford and Shah model," Int. J. Comput. Vis., V. 50, no. 3, pp. 271-293, 2002.

[10] A. Tsai, A. Yezzi, and A. Willsky, "Curve evolution implementation of the Mumford-Shah functional for image segmentation denoising, interpolation, and magnification," IEEE Trans. Image Processing, V. 10, no. 8, pp. 1169-1186, Aug. 2001.

[11] N. Paragios, and R. Deriche, "Coupled geodesic active regions for image segmentation: A level set approach," Proceedings ECCV'00, Dublin, V. II, pp. 224-240.

[12] X. Xie, and M. Mirmehdi, "RAGS: Region-aided geometric snake," IEEE Trans. Image Processing, V. 13, no. 5, pp. 640-652, May 2004.

[13] R. Kimmel, "Fast edge integration," in Geometric Level Set Methods in Imaging, Vision, and Graphics, S. Osher and N. Paragios (Ed.), Springer, pp. 59-74, 2003.

[14] V. Caselles, R. Kimmel, and G. Sapiro, "Geodesic active contours," Int. J. Comput. Vis., V. 22, no. 1, pp. 61-79, 1997.

[15] D. Mumford and J. Shah, "Optimal approximations by piecewise smooth functions and associated variational problems," Commun. Pure Appl. Math., V. 42, pp. 577685, 1989.

[16] P. Perona, J. Malik, "Scale-space and edge detection using anisotropic diffusion," IEEE Trans. PAMI, V. 12, no. 7, pp. 629-639, July 1990. 
[17] Y. You, W. Xu, A. Tannenbaum, M. Kaveh, "Behavioral Analysis of Anisotropic Diffusion in Image Processing," IEEE Trans. Image Processing, V. 5, no. 11, pp. 1539-1553, Nov. 1996.

[18] R.T. Whitaker, S. M. Pizer, "A Multi-scale Approach to Nonuniform Diffusion," CVGIP: Image Understanding, V. 57, no. 1, pp. 99-110, Jan. 1993.

[19] S. Kichenassamy, "The Perona-Malik Paradox," SIAM J. Appl. Math. Vol. 57, No. 5, pp. 1328-1342, Oct. 1997.

[20] J. Weickert, B. Benhamouda, "A semi-discrete nonlinear scale-space theory and its relation to the PeronaMalik paradox," F. Solina, W.G. Kropatsch, R. Klette, R. Bajcsy (Eds.), Advances in Computer Vision, Springer, Wien, pp. 1-10, 1997.

[21] Y. You, M. Kaveh, "Differences in the behaviors of continuous and discrete anisotropic diffusion equations for image processing," Int'l Conf. on Image Processing, V. 3, pp. 249-253, 1998.

[22] L. Alvarez, R. Lions, J. Morel, "Image Selective Smoothing and Edge Detection by Nonlinear Diffusion II," SIAM J. Numer. Anal. V. 29, no. 3, pp. 845-866, June 1992.

[23] R.A.Carmona, S. Zhong, "Adaptive Smoothing Respecting Feature Directions," IEEE Trans. Image Processing, V. 7, no. 3, pp. 353-358, Mar. 1998.

[24] J. Shah, "A Common Framework for Curve Evolution, Segmentation and Anisotropic Diffusion", Proc. CVPR, pp. 136-142, 1996.

[25] Y. Chen, B. Vemuri, L. Wang, "Image Denoising and Segmentation via Nonlinear Diffusion," Computers and Mathematics with Applications, V. 39, no. 5/6, pp. 131149, 2000.

[26] S. Teboul, L. Blanc-Féroud, G. Aubert and M. Barlaud, "Variational Approach for Edge-Preserving Regularization Using Coupled PDE's", IEEE Trans. Image Processing, V. 7, no. 3, 1998.

[27] M. Proesmans, E. Pauwels, L. Gool, "Coupled Geometry-Driven Diffusion Equations for Low-level Vision," in Geometry-Driven Diffusion in Computer Vision, Bart M. ter Haar Romeny (Ed.), Kluwer, pp. 191228, 1994.

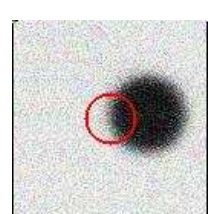

(a)

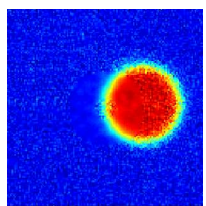

(b)

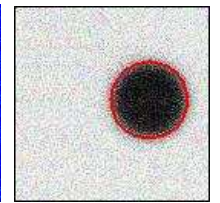

(c)

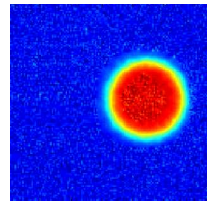

(d)
Figure 1. Region information in the model drives the curve, which is initially set to shrink, to evolve to correct position. (a) image with initial contour, size $=128 * 128$. (b) the region field at the start. (c) final segmentation result. (d) the region field in the end. ( $\alpha=0.2,44$ iterations, CPU = 6.93s).

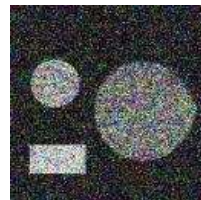

(a)

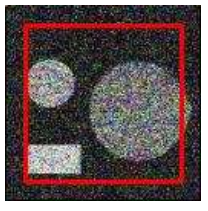

(b)

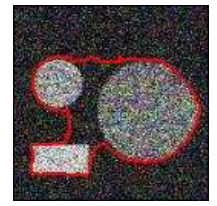

(c)

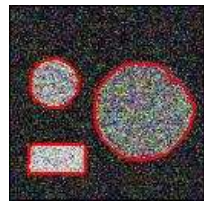

(d)
Figure 2. Segmentation of multiple objects in a noisy image. Multiple objects can be successfully segmented using the proposed model even in a very noisy image (size $=150$ * 150) $(\alpha=0.1,348$ iterations, CPU = 20.06s).

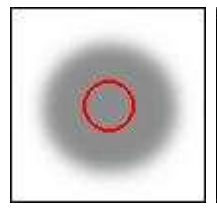

(a)

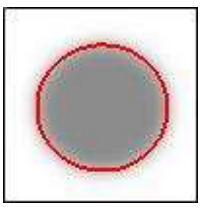

(b)

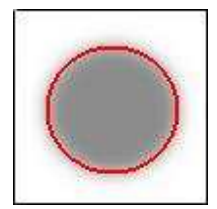

(c)

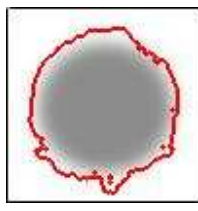

(d)
Figure 3. The boundary leakage problem. (a) image with weak edges with initial contour, size $=100 * 100$. (b) results using the proposed method $(\alpha=0.1,451$ iterations, $\mathrm{CPU}=$ 5.14s). (c) results using the method in [8] ( $\alpha$ $=0.1,51$ iterations, $\mathrm{CPU}=0.84 \mathrm{~s}$ ). (d) results using the method in [6], where boundary leakage occurs ( $\alpha=1.0,1137$ iterations, 13.32s). 


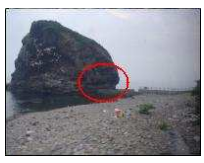

(a1)

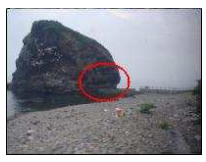

(a2)

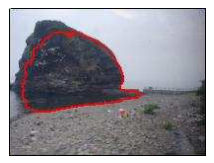

(b1)

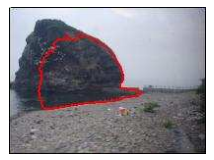

(b2)

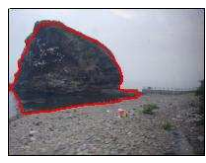

(c1)

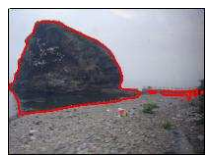

(c2)

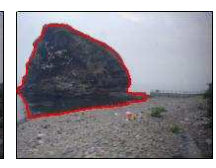

(d1)

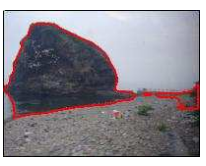

(d2)
Figure 4. Segmentation of real images. (a1)(a2) original images with initial contour, size $=200 * 150$. (b1)-(d1) results using the proposed method $(\alpha=0.2,334$ iterations, CPU $=57.99 \mathrm{~s})$. (b2)-(d2) results using the method in [8] $(\alpha=0.1,934$ iterations, CPU $=84.13 \mathrm{~s})$.

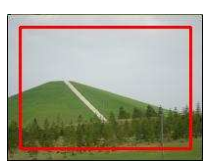

(a1)

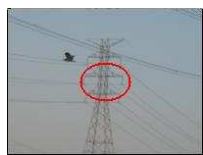

(a2)

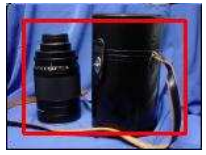

(a3)

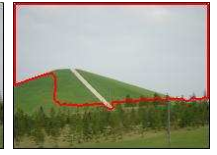

(b1)

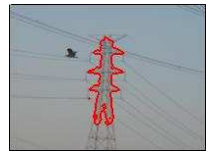

(b2)

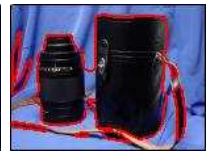

(b3)

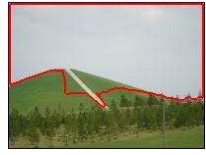

(c1)

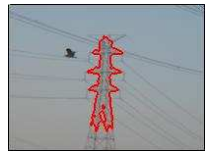

(c2)

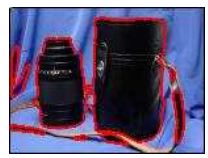

(c3)

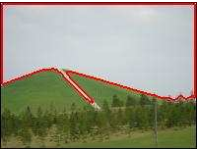

$(\mathrm{d} 1)$

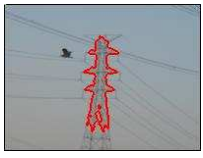

(d2)

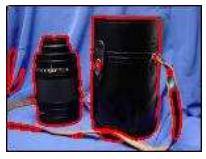

(d3)
Figure 5. Segmentation of real images. (a1)(d1) results for the image with a mountain. (size $=200 * 150, \alpha=0.2,680$ iterations, CPU $=52.53 \mathrm{~s}) .(\mathrm{a} 2)-(\mathrm{d} 2)$ results for the image with a tower $(\alpha=0.1,115$ iterations, $\mathrm{CPU}=20.56 \mathrm{~s})$. (a3)-(d3) results for image with a bag $(\alpha=0.2$,

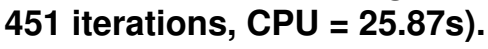

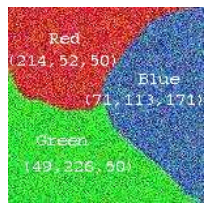

(a)

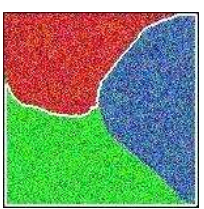

(b)

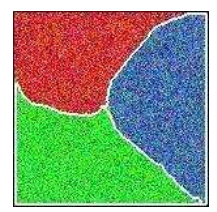

(c)

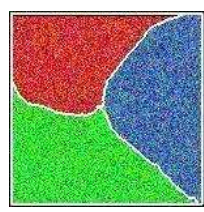

(d)
Figure 6. Segmentation of triple junctions in an image using an hierarchical approach. (a) Original image, size $=150 * 150$. (b) Segmentation results after first iteration. (c) Segmentation results after second iteration. (d) final results.

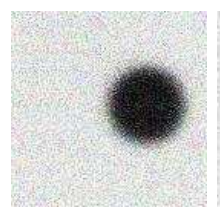

(a)

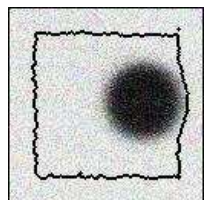

(e)

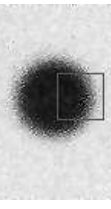

(b)

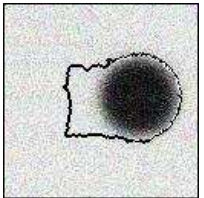

(f)

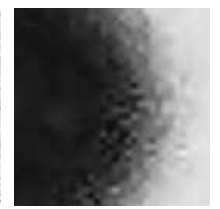

(c)

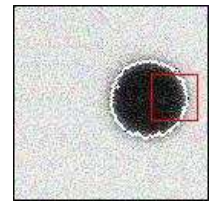

(g)

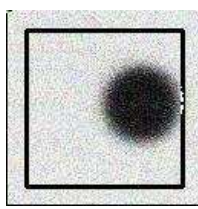

(d)

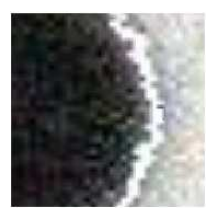

(h)

Figure 7. Solution of the staircasing problem. (a) Original image, size $=182 * 182$. (b) Staircasing problem by PM method $(K=10)$. (c) Magnified portion in (b). (d)-(g) segmentation process of the proposed model. (h) Magnified portion of $(\mathbf{g})$.

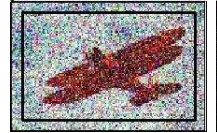

(a)

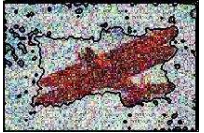

(b)

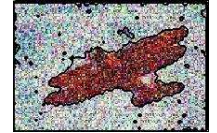

(c)

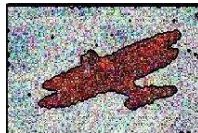

(d)

Figure 8. Segmentation of an image with heavy noise (size $=211 * 141, \alpha=0.2,289$ iterations, CPU $=193.88 \mathrm{~s}$ ). (a) Original image. (b) and (c) intermediate results. (d) final results. 